\title{
O PROBLEMA DO CARTEIRO CHINÊS APLICADO NA OTIMIZAÇÃO DAS ROTAS DE COLETA DE RESÍDUOS RECICLÁVEIS: UM ESTUDO DE CASO
}

\author{
Matheus Fernando Moro ${ }^{*}{ }^{*}$, Dalton Francisco de Andrade ${ }^{2}$, Bruno Miranda dos Santos ${ }^{3}$, Cyro Rei Prato Neto ${ }^{3}$, Juliane de Freitas \\ Battisti $^{3}$ \\ 1 Programa de Pós-Graduação em Engenharia de Produção, Universidade Federal de Santa Catarina, 88040-900, Florianópolis, \\ Brasil. \\ ${ }^{2}$ Departamento de Engenharia de Produção; Departamento de Informática e Estatística, Universidade Federal de Santa Catarina, \\ 88040-900, Florianópolis, Brasil. \\ 3 Programa de Pós-Graduação em Engenharia de Produção, Universidade Federal de Santa Maria, 97105-900, Santa Maria, Brasil.
}

*E-mail: matheus.moro@posgrad.ufsc.br

\section{RESUMO}

O Problema do Carteiro Chinês caracteriza-se pela roteirização de arcos e tem como objetivo a cobertura de arcos de um grafo, criando uma rota que passe ao menos uma vez em cada um destes arcos. Nesta pesquisa, o algoritmo do Problema do Carteiro Chinês foi aplicado na área urbana da cidade de Matelândia/PR, para otimizar a rota percorrida pelo caminhão de coleta de resíduos sólidos recicláveis. O estudo foi dividido em três partes: segunda-feira, terça-feira e quarta-feira, pois cada dia o caminhão faz uma rota diferente. Por meio do resultado do algoritmo do Carteiro Chinês utilizou-se do algoritmo de Fleury para encontrar a rota de cada um dos dias. A utilização destes algoritmos forneceu uma solução satisfatória para o problema de geração de rotas na coleta de resíduos sólidos recicláveis. Na área onde o Algoritmo do Carteiro Chinês foi aplicado, obteve-se um ganho aproximado de 23,45\%, 33,12\% e 40,13\% para segunda, terça e quarta-feira respectivamente.

Palavras-chave: Problema do carteiro chinês. Otimização de rotas. Coleta de lixo reciclável.

\section{Introdução}

A coleta de lixo no Brasil é uma tarefa de responsabilidade dos governos municipais, executada normalmente e diariamente na grande maioria das cidades brasileiras. Dados de 2012 produzidos segundo IBGE, indicam que $98 \%$ dos domicílios das zonas urbanas têm serviço de coleta de lixo, enquanto que apenas $23 \%$ dos domicílios são atendidos na zona rural. Estas marcas surpreendentes colocam o Brasil entre os maiores produtores de lixo do mundo, com dispêndio elevadíssimo da ordem de $\mathrm{R} \$ 8$ bilhões por ano. $\mathrm{O}$ custo da coleta somente com equipamentos $\mathrm{e}$ pessoal indica ser aproximadamente $50 \%$ deste total [1]. Com o crescimento da população e o consequente acréscimo do consumo de bens de alimentação per capita e de acordo com o Censo Demográfico do IGBE de 2010, 84\% da população brasileira concentra-se em áreas urbanas, ocasionando um crescente aumento do volume de resíduos produzido, apresentando assim a importância do gerenciamento de resíduos em áreas urbanas.

A preocupação por parte do poder público está estreitamente vinculada à aceitação da administração municipal por parte da população. Os serviços de limpeza absorvem entre 7\% e $15 \%$ dos recursos de um orçamento municipal, dos quais $50 \%$ são destinados exclusivamente à coleta e ao transporte de resíduos, desse modo, certamente a sua otimização leva a uma economia significativa dos recursos públicos [2].

De acordo com [3], uma empresa de coleta de resíduos residenciais precisa elaborar um método operacional que satisfaça as necessidades da população, mas que também respeite o limite de custos suportado pela empresa. Neste contexto, a pesquisa operacional e a logística, apesar de terem muito a contribuir, no Brasil ainda há resistências em empregar suas técnicas amplamente nos mais diversos setores da cadeia de produção [4; $5 ; 6]$. Parte disso deve-se ao fato de que, às vezes, os cálculos são complexos e difíceis de serem formulados, com o agravante de que há situações em que mesmo conseguindo formular corretamente o problema, a parte computacional pode demandar muito esforço, pois os carros de coleta de resíduos residenciais devem ser distribuídos ao longo da cidade e ao longo da semana de forma que nenhuma região deixe de ser atendida por um espaço de tempo muito longo. Segundo [3], algumas regiões mostram-se mais importantes do que outras sejam porque produzem maiores quantidades de lixo ou porque têm maiores valores urbanísticos, precisando ser priorizadas.

Devido ao considerado montante absorvido pela atividade de limpeza urbana, a otimização das rotas de coletas de resíduos 
recicláveis torna-se pertinente para os municípios. Essa otimização tende a ser mais expressiva em municípios pequenos, visto que a sua otimização com técnicas da pesquisa operacional é facilitada, pois sua rua em geral tem mão dupla. Entretanto percebe-se uma escassez em pesquisas focadas em municípios desse porte. É comum o uso de técnicas da pesquisa operacional para otimização de rotas de coleta de lixo, como o Problema do Carteiro Chinês (PCC), o qual foi utilizado [7] para otimizar o serviço de coleta de lixo em Joinville, obtendo um ganho de 7,83\%, já [8] utilizaram o PCC para otimizar serviço de coleta de resíduos na cidade turca de Trabzon, obtendo uma economia de $24,7 \%$ na distância da rota. [9] utilizaram o PCC para otimizar a rota de coleta de resíduos no bairro de Pinheiros, São Paulo, onde obteve 17,91\% de redução. $\mathrm{Na}$ Eslovênia e conseguiram redução de $21 \%$ na rota de coleta de lixo [10].

O que há em comum nesses estudos é que as cidades onde foi aplicado o PCC são cidades com mais de cem mil habitantes e todas estas cidades possuem várias ou algumas ruas de mãos únicas, o que de acordo com [11] impossibilita a utilização do Problema do Carteiro Chinês Não Direcionado (PCCND), tendo que ser utilizado o PCC Direcionado (PCCD). Assim, essa pesquisa alça o seguinte questionamento: É possível aplicar o método simples do PCC, o PCCND em cidades pequenas e obter bom resultado na redução das distâncias das rotas de coleta de lixo?

Sob este questionamento, a presente pesquisa tem como objetivo, minimizar a rota da coleta de lixo reciclável na cidade de Matelândia, aplicando o Problema do Carteiro Chinês (PCC), a fim de encontrar uma alternativa na diminuição do gasto que a administração municipal tem com o transporte de resíduos de lixo reciclável na área urbana da cidade.

Este artigo divide-se em quatro seções, além desta introdução. Na segunda seção apresenta a descrição do problema, posteriormente tratam-se dos procedimentos, materiais e métodos utilizados nessa pesquisa. No quarto são realizadas as análises dos resultados do trabalho. E o último apresenta as conclusões da pesquisa.

\section{Referencial Teórico}

Devido ao aumento da população mundial, surgem cada vez mais problemas de ordem pública e social que carecem de soluções eficazes. Um desses problemas, cada vez mais iminente no contexto atual, é que, ano após ano, maiores quantidades de resíduos são geradas por efeito de um crescimento da taxa de consumo [12,6]. Essas taxas influenciam na redução de matérias primas e no aumento da poluição ambiental, uma vez que se identifica uma falta de fluxo de recirculação destes fatores [13]. Diante disso, de acordo com o Instituto de investigação e proteção ambiental DEFRA, diversos países elevaram seus gastos com proteção ambiental, sobretudo, com programas de gerenciamento de resíduos [14]. Por exemplo, os países da União Europeia, em seus programas de proteção ambiental, dispenderam $40 \%$ a mais de seu orçamento no período de 2003-2013 para atividades que correspondem a gestão de resíduos. Por outro lado, no Brasil, em termos de recursos destinados pelos municípios brasileiros em 2015 para fazer frente a todos os serviços de limpeza urbana no Brasil foram, em média, de cerca de $\mathrm{R} \$ 10,15$ por habitante por mês, o que representa um pequeno aumento de $1,7 \%$ em relação a 2014, denotando que ações voltadas para melhorias nesse campo são fundamentais para o desenvolvimento do país.

As situações que englobam a problemática da gestão de resíduos são bem diferentes de município para município e desta forma acabam por atingir mais vigorosamente algumas prefeituras do que outras. O problema da limpeza pública em centros urbanos evidencia uma complexidade cada vez maior à medida que crescem os contingentes populacionais. Em virtude disso, o gerenciamento dos resíduos sólidos apresenta-se como uma atividade de ordem complexa no sentido de efetuar a coleta de maneira otimizada e adequada [15,5]. No Brasil, os números referentes à geração de Resíduos Sólidos Urbanos (RSU) denotam um total anual de 79,9 milhões de toneladas no país, demonstrando um crescimento superior ao registrado em anos anterior, de acordo com dados da Associação Brasileira de Limpeza Pública e Resíduos Especiais ABRELPE.

Em uma comparação entre as quantidades de RSU gerada e o montante que foi efetivamente coletado no ano de 2015, 72,5 milhões de toneladas, indica em um índice de abrangência de coleta de $90,8 \%$ para o país, o que reflete cerca de 7,3 milhões de toneladas de resíduos sem coleta e com destino impróprio. A população brasileira apresentou um aumento de 0,8\% entre 2014/2015 e a geração per capita de RSU cresceu no mesmo compasso. Em contrapartida, a geração total atingiu o equivalente a $218.874 \mathrm{t} / \mathrm{dia}$ de RSU gerado no país. No tocante referente a quantidade de RSU coletados em 2015, nota-se que houve um crescimento em todas as regiões do país, em comparação com o ano de 2014, conforme Figura 1. A região Sudeste, por exemplo, continua respondendo por aproximadamente $53 \%$ do total e apresenta o maior percentual de cobertura dos serviços de coleta do país [5].
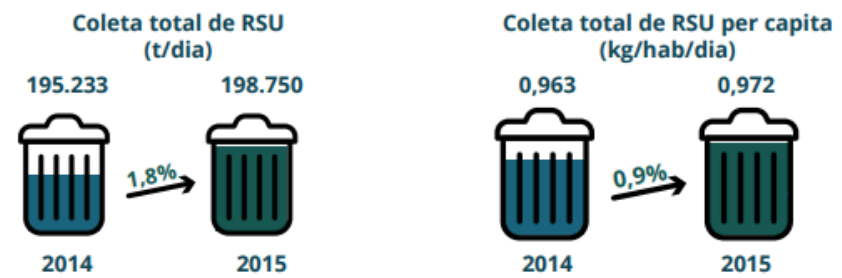

Figura 1 - Coleta de RSU no Brasil. Fonte: ABRELPE (2015)

Embora tenha se evidenciado um acréscimo, tanto da coleta total como da per capita, a região Sul, onde se localiza a cidade na qual foi desenvolvido o estudo de caso, figura apenas como a terceira região que mais contribuiu para a evolução desses números, de acordo com a Figura 2, constatando que novas 
metodologias para solução do problema do lixo podem ser utilizadas pela administração pública como forma de otimizar o processo de coleta em grandes e pequenas cidades.

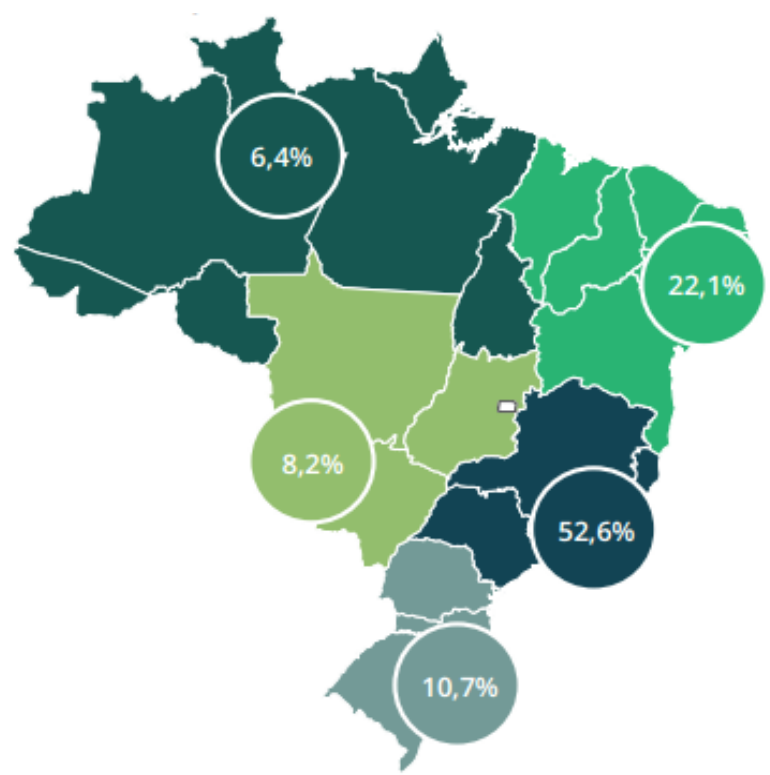

Figura 2 - Contribuição das regiões do país no total de RSU coletado. Fonte: ABRELPE (2015)

Como estímulo para o uso de metodologias para encontrar soluções para o problema da coleta de lixo, é importante ressaltar que os serviços de limpeza consomem entre $7 \%$ e $15 \%$ dos recursos de um orçamento municipal. Logo, um gerenciamento eficiente dos serviços de coleta e transporte do lixo, refletiria em uma importante contribuição para os serviços públicos. Além disso, a otimização de rotas de coleta geraria uma economia significativa do orçamento público.

Referente a coleta do lixo urbano, diversas pesquisas foram desenvolvidas ao longo dos anos [7,9,15-17]. Nesse contexto, as diversas áreas da Pesquisa Operacional têm papel fundamental na obtenção de caminhos que visem a minimização dos gastos destinados ao processo de coleta e transporte dos resíduos, propiciando uma melhor alocação dos recursos públicos designado para este fim. O problema de coleta e transporte de lixo, na literatura, podem ser solucionados por meio dos problemas de roteamento de arcos, cujo objetivo é determinar uma rota de custo mínimo em um subconjunto de arcos de um grafo, dado que todos os arcos sejam atravessados ao menos uma vez, podendo a rota ter ou não restrições específicas. Portanto, pode-se ter a intenção de rotear um veículo de cada vez ou $n$ veículos ao mesmo tempo.

Dentre os diversos problemas de roteamento em arcos podemos mencionar o Problema do Carteiro Chinês (PCC) que, classifica-se em: Problema do Carteiro Chinês Não Direcionado (PCCND), Problema do Carteiro Chinês Direcionado (PCCD) e Problema do Carteiro Chinês Misto (PCCM). No entanto, neste trabalho aborda o PCCND devido a delimitação deste estudo. A seguir, a descrição do problema onde se fez o uso do PCCND é descrita detalhadamente.

\section{Descrição do Problema}

Matelândia com seus 16078 habitantes [18] gera aproximadamente 14,5 toneladas de lixo diariamente, ou seja 0,9 $\mathrm{kg} / \mathrm{hab} /$ dia. Destas 14,5 toneladas; 8,7 toneladas são materiais recicláveis, porém, atualmente apenas 0,9 toneladas estão sendo recicladas. O restante é disposto no aterro sanitário misturado com o lixo orgânico.

Os $900 \mathrm{~kg}$ de lixo que são reciclados por dia são levados para o barracão de triagem que é o local onde os Agentes Ambientais separam os materiais recicláveis em papel, vidro, metal e plástico, enfardam e os comercializam. O local de triagem é composto por um barracão de $297 \mathrm{~m}^{2}$, com baias para a colocação dos materiais recicláveis previamente separados, prensa e balança para a comercialização desses materiais recolhidos no perímetro urbano e rural no Município de Matelândia. A coleta dos resíduos na cidade de Matelândia é efetuada por meio de um caminhão coletor-compactador de uma empresa terceirizada contratada pela própria prefeitura. É diferenciada para os diversos tipos de resíduos, sendo que os caminhões coletam somente os resíduos domiciliares e comerciais. Para os outros tipos de resíduos, tais como de saúde, industrial, agrotóxicos, oficinas e postos de combustíveis, há uma legislação específica do CONAMA para cada tipo de material, onde o gerador é responsável pela sua destinação.

Esse serviço é realizado de segunda-feira a sábado, sendo oferecido diariamente na área central devido ao grande número de residências e comércio dos diferentes ramos, sendo que nos bairros a coleta é realizada apenas uma vez por semana. A coleta também é realizada nas duas Áreas Industriais, uma vez por semana e em três distritos do município. Para o desenvolvimento da presente pesquisa será levada em conta apenas os dias que o caminhão percorre somente o período urbano, ou seja, segunda-feira, terçafeira e quarta-feira. Assim a pesquisa é composta de três estudos distintos, um para cada um dos três dias da semana em estudo.

O objetivo da prefeitura é organizar as rotas de forma a minimizar o número de viagens que o caminhão terá que percorrer até o barracão de triagem e também que ele se possível não passe duas vezes na mesma quadra, problema observado todos os dias na coleta. Atualmente, nenhum método tem sido adotado para definir o trajeto a ser percorrido pelo caminhão de coleta, a responsabilidade é dos funcionários, que com base no seu conhecimento empírico buscam a melhor e possivelmente a menor rota possível. Na Tabela 1 podem-se visualizar as distâncias percorridas pelo caminhão em cada um dos estudos. 
Tabela 1. Distâncias percorridas em cada dia de coleta

\begin{tabular}{cc}
\hline Dia da Semana & Distância percorrida $(\mathbf{k m})$ \\
\hline Segunda-feira & 35,26 \\
Terça-feira & 44,89 \\
Quarta-feira & 40,38 \\
\hline Média & $\mathbf{4 0 , 1 7}$ \\
\hline
\end{tabular}

Esses trajetos foram realizados no mês de novembro de 2016. Os trajetos têm em média $40 \mathrm{~km}$ cada, essas distâncias foram calculadas através do GPS no caminhão e depois com o auxílio do programa GPS TrackMaker mediu-se um ponto inicial de coleta até o posterior retorno ao mesmo ponto.

\section{Metodologia}

Para atingir o objetivo proposto, na Figura 3 é possível observar as etapas que compõe a pesquisa. A coleta de dados é uma parte fundamental do processo de simulação, pois o insucesso nesta etapa compromete todo o trabalho. Os dados foram coletados com a ajuda da Secretária de Secretaria Municipal de Agricultura, Meio Ambiente e Desenvolvimento Econômico de Matelândia/PR SMAMADE. Durante o mês de novembro de 2016 um funcionário da secretaria juntamente com um pesquisador colocou um GPS no caminhão de coleta para que assim pudesse encontrar as rotas do caminhão durante aquele período, pois até então o município não contava com essa informação.

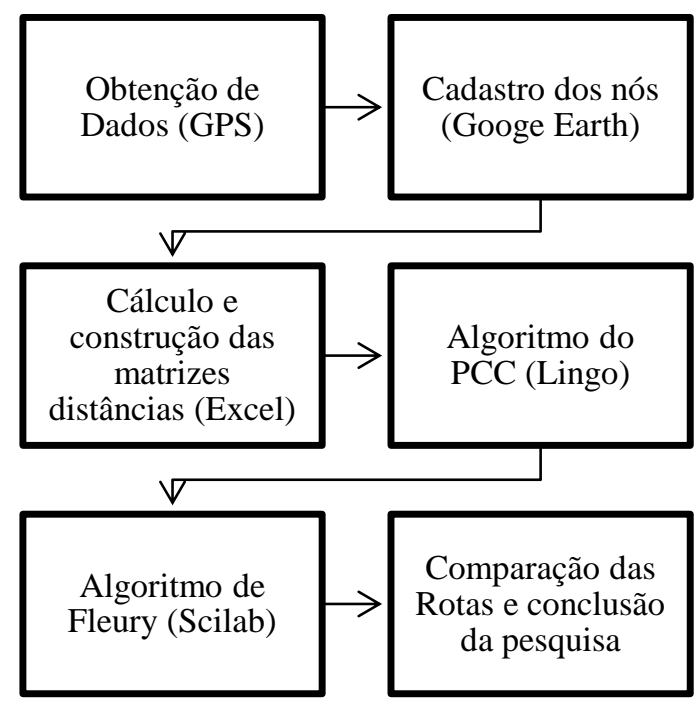

Figura 3 - Etapas da pesquisa.

O GPS colocado no caminhão contava com um aplicativo que enviava os dados coletados para um software, o GPS Tracker®. Este software permite acoplar mapas de arquivo do software AutoCad®, a partir disso, mapas do municipio alinhados com os dados puderam verificar as rotas durante cada dia da semana. Todavia o software GPS Tracker ${ }^{\circledR}$ mostra somente a rota onde o caminhão passou a cada dia, mas não fornece a distância entre os nós (esquinas), para obter isso foi preciso cadastrar cada esquina com um número e retirar as distâncias entre os nós através do Google Earth.

O Google Earth é um aplicativo desenvolvido e distribuído pela empresa que leva a logomarca Google, cuja função é disponibilizar um modelo tridimensional do globo terrestre. Este modelo é construído a partir da captura de imagens via satélite, obtidas de diversos ângulos de visão, imagens aéreas (através de aeronaves) e sistemas de informações geográficas 3D. Esse aplicativo foi utilizado para a obtenção das distâncias entre os nós cadastrados. Na Figura 4, para fim de demonstração, pode ser observado os nós cadastrados referentes à Segunda-feira. Para terça e quarta o mesmo processo foi usado.

Após o cadastramento de todos os nós/esquinas, utilizou-se o Excel para formar uma matriz distância para cada dia de estudo. Após a conclusão da construção das matrizes distâncias desenvolveu-se algoritmo do PCCND no software Lingo®. Neste algoritmo é necessário importar as matrizes distâncias para o programa e assim o algoritmo gera uma outra matriz como resultado, esta, chamada de matriz de adjacências.

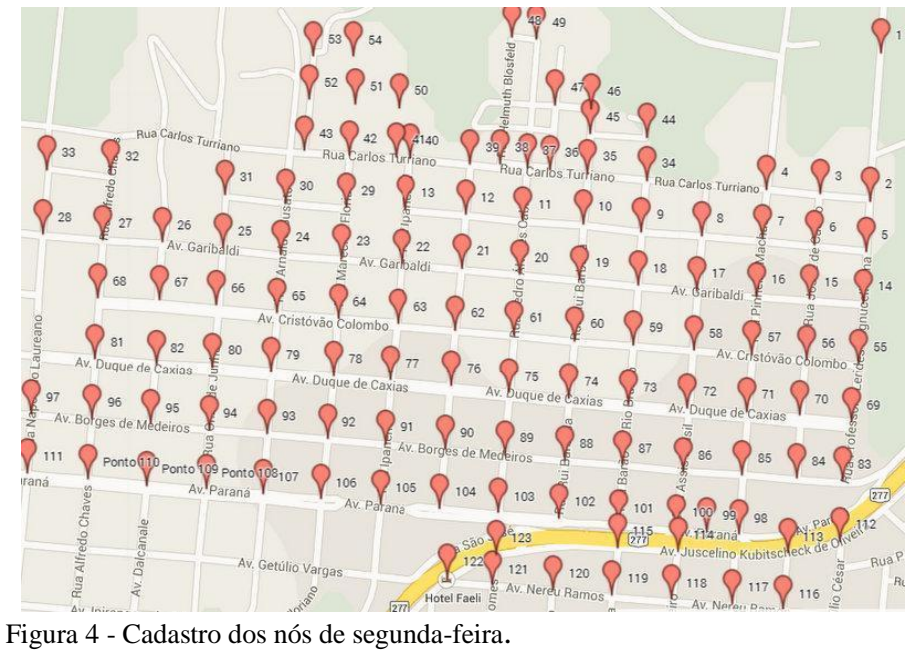

Por fïm o algoritmo de construção de rota, o Algoritmo de Fleury é programado no software Scilab 5.2.2. As matrizes de adjacências de cada dia em estudo são importadas para o programa, que quando executado gera a sequência de nós (pontos) que formaram a rota otimizada. É importante ressaltar que cada algoritmo, tanto o PCCND e de Fleury precisa ser executado três vezes, uma vez para cada estudo de caso de cada dia da semana. Tendo os resultados, é realizada uma comparação com a rota atualmente empregada pelo município e realizada as discussões pertinentes. 


\subsection{Modelo Matemático}

A cidade de Matelândia possui apenas ruas de mão dupla, portanto neste trabalho será utilizado o PCCND para determinar a rota mínima de coleta de lixo reciclável no município. Segundo [19], Kwan Mei-Ko foi o primeiro a relatar PCCND em uma publicação datada de 1962 no periódico "Chinese Mathematics". Em virtude de sua origem, o problema ficou denominado como Problema do Carteiro Chinês. A formulação matemática do PCCND, apresentada por [15], é apresentada a seguir:

\section{Minimizar}

$$
\sum_{i=1}^{n} \sum_{j=1}^{n} c_{i j} x_{i j}
$$

Sujeito a:

$$
\begin{aligned}
\sum_{k=1}^{n} x_{k i}-\sum_{k=1}^{n} x_{k i} & =0 \quad i=1, \ldots, n \\
x_{i j}+x_{j i} & \geq 1 \quad \forall(\mathrm{i}, \mathrm{j}) \in A \\
x_{i j} & \geq 0 \text { inteiras }
\end{aligned}
$$

onde:

$x_{i j}=$ número de vezes em que a aresta $(i, j)$ é percorrido de $i$ para $j$

$c_{i j}=$ comprimento ou o custo da aresta $(i, j)$.

No modelo matemático proposto, a função objetivo (1) minimiza o custo total, ou seja, no caso do trabalho em estudo, a distância total a ser percorrida. As restrições em (2) garantem a continuidade da rota, as restrições em (3) que nenhuma aresta deixará de ser considerada e, em (4), tem-se que as variáveis do problema são não negativas, inteiras. A solução exata deste problema pode ser obtida em $\mathrm{O}\left(\mathrm{n}^{3}\right)$ como mostra Papadimitriou [20]. Edmonds e Johnsn [21] apresentam um interessante algoritmo para a solução do PCC via matching (emparelhamento). Pode-se resumir o algoritmo da seguinte forma [20]:

\section{INICIO}

Ler o grafo $G(N, A)$ Se todos os nós em $G$, o grafo original, possuem grau par então

determinar um ciclo Euleriano em $G$ e Fim.

Organizar um grafo $K_{n}$ da seguinte forma:

Reunir todos os vértices de grau ímpar no grafo $K_{n}$ e associar a cada par de vértices $i$ e $j$ no grafo, uma aresta $(i, j)$ com peso igual ao caminho mais curto que liga $i$ a $j$ no grafo $G$.

Determinar o 1-matching mínimo em $K_{n}, M^{*}$. Para cada aresta pertencente a $M^{*}$ em $G$ no caminho mínimo que ela representa, obtendo um grafo $G_{n}$.

Determinar a solução do carteiro chinês que é representada por um ciclo Euleriano em $G_{n}$.

FIM

\subsection{Algoritmo de Construção de Rota}

Segundo Feiteira [22] se G é um grafo euleriano, então qualquer trilha fechada euleriana de $\mathrm{G}$ é uma trilha ótima, já que uma trilha fechada euleriana é uma trilha fechada que atravessa cada aresta exatamente uma vez. O Problema do Carteiro Chinês pode ser facilmente resolvido neste caso, já que existe um bom algoritmo para determinar uma trilha fechada euleriana em um grafo euleriano. $\mathrm{O}$ algoritmo de Fleury constrói uma trilha fechada euleriana pela escolha de uma aresta a cada passo, sujeita a condição de que uma aresta de corte, do subgrafo aresta induzido pelas arestas que não estão nesta trilha, é escolhida somente se não há outra alternativa.

O algoritmo de Fleury é geralmente usado quando se tem o grafo e assim ele é descrito:

1. Escolher um vértice qualquer para iniciar.

2. Escolher qualquer aresta que saia desse vértice só escolhendo uma aresta que seja um istmo se não houver mais nenhuma.

3. Destruir a aresta utilizada

4. Repetir 2 e 3 até chegar ao vértice inicial.

5. Se não há mais arestas o circuito de Euler está encontrado. Caso ainda haja arestas, recomeçar o circuito a partir de um dos vértices do circuito onde ainda haja arestas incidentes.

Este algoritmo é também de fácil programação em computador, a partir da matriz de adjacência, que é o caso da presente pesquisa. Um programa de computador tem que fazer uma escolha sistemática. Suponha-se que o programa é construído de modo que a aresta escolhida em cada passo é a correspondente à primeira entrada não nula da linha correspondente ao vértice que está a ser considerado no momento.

Função Fleury:

$(\mathrm{G}=(\mathrm{V}, \mathrm{E})$ : grafo $):$ caminho

$\mathrm{G}^{\prime}:=\mathrm{G}\left\{\mathrm{G}^{\prime}=\left(\mathrm{V}^{\prime}, \mathrm{E}^{\prime}\right)\right\} \mathrm{v} 0:=$ um vértice de $\mathrm{G}^{\prime}$

$\mathrm{C}:=[\mathrm{v} 0]\{$ Inicialmente, o circuito contém só v0 $\}$ Enquanto E' não vazio

$\mathrm{v}_{\mathrm{i}}:=$ último vértice de $\mathrm{C}$ Se vi tem só uma aresta incidente

$a_{i}:=a$ aresta incidente $a v_{i}$ em G'

Senão

$\mathrm{a}_{\mathrm{i}}:=$ uma aresta incidente a vi em G' e que não é uma ponte

Retirar a aresta $a_{i}$ do grafo $G^{\prime}$ Acrescentar $a_{i}$ no final de $C$

$\mathrm{v}_{\mathrm{j}}:=$ vértice ligado a $\mathrm{v}_{\mathrm{i}}$ por $\mathrm{a}_{\mathrm{i}}$

Acrescentar $\mathrm{v}_{\mathrm{j}}$ no final de $\mathrm{C}$

Retornar C

associar uma nova aresta 


\section{Resultados}

\subsection{Desenvolvimento Computacional}

O algoritmo do Problema Carteiro Chinês para redes não direcionadas foi desenvolvido com o objetivo de resolver o problema de cobertura de arcos (ruas de mão dupla). Primeiramente, o PCCND foi modelado e enviado ao software LINGO ${ }^{\circledR}$ para a resolução, assim tendo como resultado uma matriz de adjacências. A partir do resultado obtido no LINGO® encontrou-se a rota para cada aplicação usando-se o algoritmo de Fleury, implementado no software Scilab 5.2.2.

\subsection{Análise dos Resultados}

Os resultados obtidos para a modelagem do PCCCD foram organizados na Tabela 2. Para melhor visualização e interpretação dos resultados é realizada uma comparação com a rota atual empregada pela SMAMADE.

\begin{tabular}{cccc}
\multicolumn{4}{l}{ Tabela 2. Comparação entre distâncias atuais e otimizadas. } \\
\hline $\begin{array}{c}\text { Dia da } \\
\text { semana }\end{array}$ & $\begin{array}{c}\text { Distância } \\
\text { Otimizada }(\mathbf{k m})\end{array}$ & $\begin{array}{c}\text { Distância } \\
\text { Atual }(\mathbf{k m})\end{array}$ & $\begin{array}{c}\text { Economia } \\
(\mathbf{\%})\end{array}$ \\
\hline $\begin{array}{c}\text { Segunda- } \\
\text { feira }\end{array}$ & 26,99 & 35,26 & 23,45 \\
$\begin{array}{c}\text { Terça- } \\
\text { feira }\end{array}$ & 30,03 & 44,90 & 33,12 \\
$\begin{array}{c}\text { Quarta- } \\
\text { feira }\end{array}$ & 24,17 & 40,38 & 40,14 \\
\hline Média & $\mathbf{2 7 , 0 6}$ & $\mathbf{4 0 , 1 8}$ & $\mathbf{3 2 , 2 4}$ \\
\hline
\end{tabular}

Esse resultado foi bastante expressivo por que geralmente os municípios pequenos não possuem nenhum instrumento de controle de rotas para as coletas de lixos, como é o caso do município em estudo, o motorista tinha conhecimento que precisava passar em tais quadras, mas não tinha nenhuma rota especifica para seguir e então ele passava inúmeras vezes em algumas quadras (grafos), sem a necessidade.

Por meio do PCC, aperfeiçoa-se a rota para passar o mínimo de vezes em cada quadra, apenas algumas quadras tiveram que ser percorridas mais que uma vez, visto que algumas vias são do tipo: sem saída.

Na segunda-feira e terça-feira o caminhão é obrigado a ir mais que uma vez no barracão de coleta de lixo, isso se deve que no final de semana a um acúmulo de lixo que só é recolhido nos primeiros dias da semana e também por que nos dois primeiros dias da semana o caminhão percorre regiões maiores que na quarta-feira, assim o caminhão tem sua capacidade preenchida antes de terminar a rota do dia, tendo que ir até o barracão duas vezes nestes dias.

Por meio da Tabela 2 pode-se perceber um resultado bastante significativo com o uso do PCC para otimizar as rotas dos três estudos. Na Segunda-feira, a otimização resultou na economia de $23,45 \%$ da rota, resultando em 8269 metros a menos que a rota atual empregada para este dia. Na Terça-feira a economia foi de $33,12 \%$, equivalente a 14867 metros. Na Quarta-feira o resultado foi ainda mais surpreendente, chegou a uma economia de 40,13\%, cerca de 16206 metros a menos que a rota percorrida pela SMAMADE para este dia. Na Tabela 3 observa-se a comparação por períodos.

Analisando semanalmente o estudo podemos verificar um resultado bastante expressivo, atualmente o caminhão faz 120,54 km nos três dias de estudo, por meio da otimização via PCC essa distância fica em $81,19 \mathrm{~km}$, um ganho de $32,64 \%$.

Tabela 3. Análise da economia por períodos

\begin{tabular}{cccc}
\hline Períodos & $\begin{array}{c}\text { Distância } \\
\text { Atual } \mathbf{( k m )}\end{array}$ & $\begin{array}{c}\text { Distância } \\
\text { Otimizada }(\mathbf{k m})\end{array}$ & $\begin{array}{c}\text { Diferença } \\
(\mathbf{k m})\end{array}$ \\
\hline Semana & 120,54 & 81,19 & 39,35 \\
Mês & 482,16 & 324,76 & 157,40 \\
Ano & 5785,92 & 3897,12 & 1888,80 \\
\hline
\end{tabular}

Mensalmente o resultado fica ainda mais notável, levando em consideração que cada mês tem quatro semanas, durante o mês o caminhão faz $482,16 \mathrm{~km}$, aproximadamente. Usando a otimização por meio do PCC, a redução mensal é de cerca de 157 $\mathrm{km}$. Anualmente a diferença entre a rota atual e a otimizada chega a $1888 \mathrm{~km}$.

Essa distância é aproximadamente equivalente a que o caminhão percorre por quatro meses, ou seja, aplicando as rotas otimizadas, a SMAMADE estaria economizando quatro meses de trabalho, diminuindo as distâncias percorridas, otimizando o tempo de coleta de lixo e consequentemente a diminuição da fadiga dos funcionários e economia de combustível do caminhão.

Diante das pesquisas encontradas na literatura, as quais utilizaram o PCC para otimizar a rota de coleta de lixo, nenhum obteve um resultado tão expressivo quanto este, o que pode demonstrar que cidades pequenas tem maior potencial de otimização de rotas pelo fato de poucas possuírem ruas de mão única, na sua grande maioria são compostas de ruas de mão duplas.

\section{Conclusão}

Considerando os resultados encontrados pode-se dizer que o objetivo dessa pesquisa foi em sua totalidade alcançado, pois a metodologia desenvolvida foi implementada e foram obtidos resultados adequados na otimização das rotas do caminhão de coleta de resíduos sólidos nos três dias de estudos.

Verificou-se que a empresa necessita, além de um procedimento otimizado, também tecnológico (por exemplo, a instalação de GPS nos veículos com uso diário) na coleta de resíduos sólidos, que oriente e acompanhe o trajeto de saída, o percurso para o barracão e o retorno do caminhão à garagem. Com a implantação do Algoritmo do PCC, pôde-se obter um ganho aproximado de $23,45 \%$ na rota de segunda-feira, $33,12 \%$ na rota de terça-feira e $40,14 \%$ na rota de quarta-feira em relação ao percurso total gasto atualmente. 
O comparativo com os dados da coleta de resíduos sólidos, fornecidos pela SMAMADE, com os resultados alcançados, mostrou que as técnicas da área de Pesquisa Operacional utilizadas são uma alternativa para a redução das distâncias. A problemática da coleta de resíduos sólidos deve ser encarada de maneira multidisciplinar pelas conotações sociais, econômicas, culturais e de políticas sustentáveis necessárias. Portanto, as propostas técnicas de meios que levem a uma redução nos custos operacionais devem ser consideradas. Além disso, deverá estar preparada para acompanhar o dinamismo das cidades, pois, ao mesmo tempo em que as cidades crescem, em população e extensão, passam constantemente por modificações de suas redes viárias. Para atender a está dinâmica, os setores responsáveis por estes serviços necessitam de respostas rápidas e confiáveis. Ao final, o que se espera é um serviço de maior qualidade para a população e que ao mesmo tempo onere menos o orçamento do município.

Se as metodologias desenvolvidas forem aplicadas à cidade de Matelândia/PR, pode-se conseguir uma redução nos custos, o que pode gerar uma economia significativa para a prefeitura municipal. Menor distância percorrida pelo caminhão de coleta de resíduos sólidos proporciona redução na manutenção e despesas com combustível dos veículos. Com a otimização é possível também uma melhor alocação do pessoal, podendo inclusive reduzir o número de funcionários, envolvidos nessas atividades podendo dessa forma direcioná-los para outras competências.

Por fim, diante do questionamento que se levantou na introdução da pesquisa, pode-se auferir que é possível utilizar o PCCND em cidades de pequeno porte e seu resultado é bastante expressivo.

\section{THE PROBLEM OF THE CHINESE POSTMAN APPLIED IN THE OPTIMIZATION OF THE ROUTES OF COLLECTION OF RECYCLABLES: A CASE STUDY}

\footnotetext{
ABSTRACT: The Chinese Postman Problem is characterized as all the routing in the arcs graph which creates at least one rout that passes through all arcs at least once. In this research, the algorithm of the Chinese Postman Problem was applied in the urban region of Matelandia/PR to optimize the rout of a truck collecting recyclable solid waste. The study was divided in three parts based on different weekdays: Mondays, Tuesdays and Wednesdays; because each day had a different route. Using the results generated by the Chinese Postman Problem's algorithm, the Fleury Algorithm found one route for each day. The utilization of these algorithms provided a good solution for the problem of route generation for collecting recyclable solid waste. In the study region where the Chinese Postman was applied, the gains were approximately 23,45\%, 33,12\% and 40,13\% for Mondays, Tuesdays and Wednesdays respectively.
}

Keywords: Chinese Postman Problem. Route Optimization. Recyclable waste collection.

\section{Referências}

[1] BRASIL. Gestão de resíduos no Brasil: uma visão geral. Associação Brasileira de Empresas de Limpeza Pública e Resíduos Especiais. Disponível em: <http://www.abrelpe.org.br/noticias_detalhe.cfm?NoticiasID=2091〉. 2015. Acesso em: 21 jun, 2017

[2] DETOFENO, T.; STEINER, M. Otimização das Rotas de Coleta de Resíduos Urbanos utilizando Técnicas de Pesquisa Operacional. Anais do XXXII Congresso Nacional de Matemática Aplicada e Computação, Cuiabá, Mato Grosso, 2009.

[3] SOUZA, B. C. S.; RANGEL, L. A. D. Determinação de rota ótima de um caminhão de coleta de resíduos para um bairro baseado no problema do carteiro chinês. Anais do Simpósio de Excelência em Gestão e Tecnologia, Resende, Rio de Janeiro, 2009.

[4] ARAÚJO, C. E. G. Algoritmos genéticos híbridos sem delimitadores de rotas para problemas de roteirização de veículos. $77 \mathrm{f}$. Dissertação (Mestrado em Engenharia) - Departamento de Engenharia de Transportes da Escola Politécnica, USP, São Paulo, 2008.

[5] MINISTÉRIO DO MEIO AMBIENTE. Brasil Planos de gestão de resíduos sólidos: manual de orientação, Brasília, 2012. ISBN: 978-85-99093-21-4.

[6] PORTAL BRASIL. Em oito anos a quantidade de lixo destinada passou de $35 \%$ para 48\%. Reportagem de 2014. Disponível em: http://www.brasil.gov.br/meio-ambiente/2010/10/em-oito-anos-a-quantidade-delixo-destinada-corretamente-passou-de-35-para-58>. Acesso em: 02 dez. 2016.

[7] DETOFENO, T. C. Otimização de rotas e coleta de resíduos sólidos urbanos, utilizando técnicas de Pesquisa Operacional. Dissertação (Mestrado em Métodos Numéricos). Programa de Pós-Graduação em Métodos Numéricos em Engenharia, Universidade Federal do Paraná, Curitiba, 2009.

[8] APAYDIN, O.; GONULlU, M. T.; Route optimization for solid waste collection: Trabzon (Turkey) case study. Global NEST Journal, v. 9, n. 1, 2007.

[9] RIGONATTI, A.; RODRIGUES, J. A. V.; BAtistA, M. J. N. Gomes Experiências com coleta de lixo domiciliar e aplicação de modelos do problema do carteiro chinês misto: estudo de caso no Jardim Europa. Revista Engenharia, ano 70 , ed. 6,2012

[10] BECAJ, D.; ZEROVNIK, J.; Optimization of waste collection in maribor. Proceedings of the 10th International Symposium on Operational Research, Nova Gorica, Eslovênia, 2009.

[11] EISELT, H. A.; GENDREAU, M.; LAPORTE, G. Arc-routing problems, Part 2: The Rural Postman Problem. Operations Research, v. 43, p. 399-414. 1995.

[12] HOORNWEG, D.; BHADA-TATA, P.; KENNEDY, C. Waste production must peak this century. Nature, v. 502, p. 615-617, 2013

[13] KOPPELAAR, R., WIEKARD, H. Asessing phosphate rock depletion and phosphorus recycling options. Global Environ. Chang, v. 23, n. 6, p.1454-1466, 2013 .

[14] DEFRA. Digest of Waste and Resource Statistics - 2015 Edition. Department ofr Environment Food \& Rural Affairs. Disponível em: <www.gov.uk/government/collections/waste-and-recycling-statistics〉. 2016. Acesso em: 21 jun, 2017

[15] BODIN, L.; BELTRAMI, E.J. Networks and vehicle routing for municipal waste collection, Networks, v. 4, p. 65-94, 1974. 
.

[16] NEGREIROS, M. J.; MACULAN, N.; CLEIMAN, D. F. Optimizing Routes in Household Refuse. Collection in Rio de Janeiro (A Field Application Using SisRot-UFRJ, COPPE - Eng. de Sistemas, Rio de Janeiro-RJ, Brasil. 1991.

[17] SHAFAHI, A.; HAGHANI, A. Generalized Maximum Benefit Multiple Chinese Postman Problem. Transportation Research Part C: Emerging Technologies, v. 55, p. 261-272, 2015.

[18] IBGE. Domicílios particulares ocupados, por situação e localização da área, segundo os municípios, 2017. Disponível em: <http://www.censo2010.ibge.gov.br/sinopse/index.php?dados=212\&uf=41 Acesso em: 21 de Jan. 2017.

[19] GOLDBARG, M. C. Otimização combinatória e programação linear: modelos e algoritmos. 2 ed. Rio de Janeiro: Campus, 2005.

[20] PAPADIMITRIOU, C. H. Combinatorial Optimization Algorithms and Complexity. Nova York: Prentice Hall, 1992.

[21] EDMONDS, J.; JOHNSN, E. L. Matching, Euler Tours and the Chinese Postman Problem. Mathematical Programming , v. 5, p. 88-124, 1973.

[22] FEITEIRA, R. G. C. Grafos para todos sobre o desenvolvimento da Teoria de Grafos. 2007. 50 f. Apostila de Matemática, Universidade de Algarve, Faro, Portugal, 2007. 